\title{
A NEW CONSUMER PROFILE DEFINITION METHOD BASED ON FUZZY TECHNOLOGY AND FUZZY AHP
}

\author{
Junjie Zhang ${ }^{1}$, Xianyi Zeng ${ }^{1,2}$, Min Dong ${ }^{1}$, Hua Yuan ${ }^{3, *}$ \\ 1 Hubei Key Laboratory of Digital Textile Equipment, Engineering Research Center of Hubei Province for Clothing Information, Wuhan Textile University, \\ 430073 Wuhan, China \\ 2 GEMTEX Laboratory, ENSAIT, Roubaix, France \\ 3 Wuhan Textile and Apparel Digital Engineering Technology Research Center, School of fashion, Wuhan Textile University, 430073 Wuhan, China \\ ${ }^{*}$ Corresponding author. E-mail: 2019009@wtu.edu.cn
}

\begin{abstract}
:
The research in this paper aims to set up a new consumer profile definition method based on fuzzy technology and fuzzy AHP. The result of the study could be applied to garment recommendation systems for a special consumer. Consumer profiles are chosen as research objects. The fuzzy technology and fuzzy AHP are applied in this research, which aims to provide a new method of using fuzzy technology and fuzzy AHP to define consumer profiles. We define tall-short and fat-thin by fuzzy technology and set up the weights of consumer profile by fuzzy AHP methods. The fuzzy technology and fuzzy AHP are applied for building consumer profiles that can be used for a consumeroriented intelligent garment recommendation system.
\end{abstract}

\section{Keywords:}

Fuzzy AHP, fuzzy comprehensive evaluation method, consumer profile

\section{Introduction}

Consumer profiles can take into account a number of possibilities and tend to tell a story about a potential consumer, analyzing lifestyles, attitude, occasion of use for a product, income, age, and any other defining characteristics. This helps to target advertising and marketing to these potential consumers and cut costs.

Consumer profiles are a way of describing a consumer directly so that they can be grouped for marketing purposes [1]. To achieve a better understanding of consumer profiles, some important factors should be considered.

Geographic Segmentation: This can refer to country regions, city, and the effect of weather. Fashion is traditionally organized by season, but weather patterns at a given time of year, in holiday regions, for example, will have a strong bearing on the type of garment required.

Psychographic Segmentation: This is expressed by the lifestyle of individuals, including their activities, interests, and opinions.

Demographic Segmentation: This can refer to gender, occupation, marital status, income, wealth, education, religion, age, height, youth subcultures, region, country, climate, and so on.

Behavioral Segmentation: The final use of the product, brand loyalty, and consumer needs for certain benefits and price sensitivity are considerations.
Socioeconomic Classification: This is considered an old system now as it is based on class, but does allow for broad judgments based on occupations and often is used as a reference to the lifestyle of the consumer.

For simplicity, in this paper, we define the consumer profile as the combination of three parts (see Figure 1), such as body data, style keywords, and visual images. In this paper, brand effects and cost are not considered.

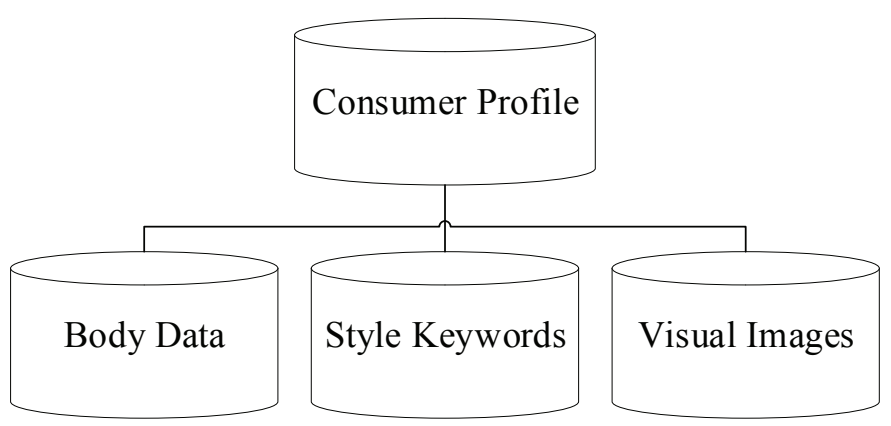

Figure 1. The proposed consumer profile.

\subsection{Body data}

Body data generally include different body measurements, such as stature, neck circumstance, chest circumstance, waist circumstance, hip circumstance, length of arm, and length of leg. Weight is also considered in some applications. 
In this paper, for simplicity, we only consider four parts, i.e. stature, weight, chest circumstance, and waist circumstance. For general consumers, these data are easier to obtain by themselves.

\subsection{Style keywords}

The method of selecting the style keywords is through sensory evaluation technology [2]. To understand each style word, we choose one reference picture from several fashion garment websites, and the Levis' official website is considered to be the most relevant one to this style word.

\subsection{Visual images}

For untrained general consumers, style keywords cannot cover all their expectations and preferences. The evaluation of the images is relatively more intuitive and closer to their perceptions of the garment products. In this paper, for the sake of simplicity, we have selected six pictures of visual images so that each consumer can choose the best image according to their own expectations.

Section 2 gives an introduction on mathematical methods, fuzzy comprehensive evaluation method (FCEM), and fuzzy AHP. Section 3 describes how to implement consumer profile by fuzzy description of height and fuzzy description of fatthin. Section 4 presents the details of a real case. Section 5 concludes the paper.

\section{Mathematical method}

\subsection{Fuzzy comprehensive evaluation method}

The FCEM is a multi-criteria comprehensive evaluation method based on fuzzy mathematics [3]. This comprehensive evaluation method converts a qualitative evaluation problem into a quantitative evaluation problem by applying operations on fuzzy sets and fuzzy relations. It is an overall hierarchical and systematic evaluation of one or several objects with respect to a number of qualitative, fuzzy, and nondeterministic criteria.

(1) Mathematical model for fuzzy comprehensive evaluation

Let $\mathrm{C}=\left\{c_{1}, c_{2}, \cdots, c_{m}\right\}$ be a set of evaluation criteria and $\mathrm{V}=\left\{v_{1}, v_{2}, \cdots, v_{n}\right\}$ a set of evaluation scores. The fuzzy relation between the universe of evaluation criteria and that of evaluation scores can be represented by the matrix $\mathrm{R}$ :

$\mathrm{R}=\left[\begin{array}{cccc} & & & \\ r_{11} & r_{12} & \cdots & r_{1 n} \\ r_{21} & r_{22} & \cdots & r_{2 n} \\ \vdots & \vdots & \ddots & \vdots \\ r_{m 1} & r_{m 2} & \cdots & r_{m n}\end{array}\right]$ $r_{i j}=\eta\left(c_{i}, v_{i}\right)\left(0 \leq r_{i j} \leq 1\right)$ presents the membership degree of the score $v_{i}$ related to the criterion $c_{i}$; the ith row in the matrix $\mathrm{R}_{i}=\left(r_{i 1}, r_{i 2}, \cdots, r_{i n}\right)$ is the distribution vector of evaluation scores on the ith evaluation criterion $c_{i}$ provided by a number of evaluators, and it is a fuzzy subset defined on $\mathrm{V}$.

\section{Determination of membership functions}

In practice, the membership functions of fuzzy sets are usually determined by professional experts having mastery over the specific context. They can also be computed using the methods of data analysis, such as fuzzy statistics, fuzzy distribution, and so on [4].

\section{Determination of weights of criteria}

There are many methods for determining importance of each evaluation criterion. These methods include determination by experts according to their direct experiences, investigative statistics, AHP by qualitative comparisons of evaluation criteria pairs, and so on. Due to the complexity, irreversibility, and fuzziness of the garment recommendation system, application of precise mathematical models will make the weights of evaluation criteria too sensitive and less robust.

In this situation, human judgments based on experts' experiences could lead to more reliable, more interpretable, and more robust results. In this research, we suggest that fuzzy AHP be used to determine the weights of evaluation criteria. It enables to determine the weights by combining three human judgments based on their experts' experiences and fuzzy operations on judgment matrices.

\subsection{Fuzzy AHP}

AHP is one of the most important methods for evaluating weights for evaluation criteria [5]. As a system decision analysis method proposed by famous US operations researcher Thomas L. Saaty in 1973, it combines both quantitative and qualitative analysis and makes full use of humans analysis, judgment, and comprehensive abilities, and is thus applicable to decision problems that have complex structures, multiple decision rules, and are not easily quantified. It can decompose, according to the predefined overall objectives, the problem into several criteria on the perspective of the system, construct a layered structure model from its dominance relations, and then apply pairwise comparison to determine relative importance between decision schemes, thus avoiding the randomness of determining weights by humans and allowing the evaluation results to be more objective and reasonable. Thus, a satisfactory decision effect can be achieved.

As proposed by Dutch scholar Van Loargoven in 1983, fuzzy AHP is an extension of AHP under fuzzy conditions. Fuzzy AHP is a method that, on the basis of comprehensive evaluation in fuzzy mathematics, makes quantitative analysis of nonquantitative events by establishing fuzzy consistent matrix with AHP-based methods for constructing weight sets; also, fuzzy AHP is a means for making an objective description 
of a human's subjective judgment. The calculation of the weight distribution of evaluation variables with fuzzy AHP can effectively reduce the subjectivity of the evaluation criteria. The difference between fuzzy AHP and AHP lies in the fuzziness of judgment matrix. The core of fuzzy AHP is to construct a judgment matrix by comparison of evaluation criteria pairs with triangular fuzzy numbers (TFNs). It allows the decision to be more reasonable by overcoming the drawbacks in the AHP methods.

\subsection{Implementation steps}

Users of the AHP first decompose their decision problem into a hierarchy of more easily comprehended subproblems, each of which can be analyzed independently. The elements of the hierarchy can relate to any aspect of the decision problemtangible or intangible, carefully measured or roughly estimated, well or poorly understood-anything at all that applies to the decision at hand.

Once the hierarchy is built, the decision makers systematically evaluate its various elements by comparing each pair of them at a time, with respect to their impact on an element above them in the hierarchy. During the comparisons, instead of using specific data about the elements, the decision makers typically integrate their judgments on relative importance values into the computation.

The procedure of AHP converts the human evaluations into numerical values that can be processed and compared over the entire range of the problem. A numerical weight or priority is derived for each element of the hierarchy, allowing diverse and often incommensurable elements to be compared in pairs in a rational and consistent way. This capability distinguishes the AHP from the other decision-making techniques.

In the final step of the process, the numerical priorities are calculated for each of the decision alternatives. These numbers represent the alternatives' relative ability of achieving

the decision goal, and thus they allow a straightforward consideration of the various courses of action.

The AHP decomposes complex problems into multiple criteria and makes up hierarchical structures according to the dominance relation between these criteria. The relative importance of various criteria is identified by using pairwise comparison judgment and sorted by synthesizing the judgment of deciders.

The basic steps of the AHP modeling are given as follows.

Step 1: Establishment of a hierarchical structure model

The hierarchical structure model is composed of the target layer, the criterion layer, and the schematic layer. The target layer is the uppermost layer, and in this layer, there is a criterion for expressing the purpose of decision and the problem. The criterion layer (middle layer), also called restraint layer or index layer, expresses the criteria and measure for realizing an object. It can be resolved into sub-criterion layers if there are too many criteria. The schematic layer (lowermost layer), also called object layer, expresses the alternatives or solutions of the decision. A typical hierarchical structure is shown in Figure 2.

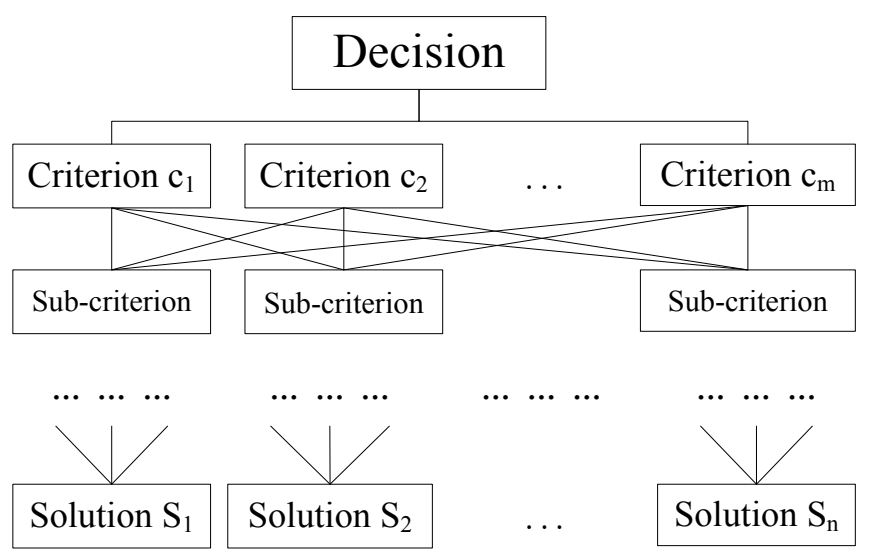

Figure 2. A typical hierarchical structure.

Table 1. The comparison between two criteria at the same level.

\begin{tabular}{|c|c|}
\hline Scores $a_{i j}$ & Implication \\
\hline 1 & The importance values of the criteria $c_{i}$ and $c_{j}$ are equal \\
\hline 3 & The criterion $c_{i}$ is a little more important than the criterion $c_{j}$ \\
\hline 5 & The criterion $c_{i}$ is more important than the criterion $c_{j}$ \\
\hline 7 & The criterion $c_{i}$ is much more important than the criterion $c_{j}$ \\
\hline 9 & The criterion $c_{i}$ is extremely more important than the criterion $c_{j}$ \\
\hline $2,4,6,8$ & The medium values of the above judgments \\
\hline Reciprocal & $\begin{array}{l}\text { If the ratio of importance of the criterion } c_{i} \text { related to } c_{j} \text { is } a_{i j} \text {, then the ratio of importance of } \\
\text { the criterion } j \text { related to } i \text { is } a_{j i}=1 / a_{i j}\end{array}$ \\
\hline
\end{tabular}


Step 2: Construction of the pairwise comparison matrix

Generally, the weights of various criteria are not always identical in the process of measuring target. If the number of criteria is too large, it is difficult to assign weights to the criteria directly. Thomas presented a Coincident Matrix Method, also called Pairwise Comparison Method. This method uses relative scores for reducing the difficulty when comparing criteria with different natures and increasing the accuracy of the weights.

Let $a_{i j}(i, j \in\{1,2, \cdots, m\})$ be the relative importance (good or bad preference) score of the $i$ th criterion $c_{i}$ related to $j$ th criterion $c_{j}$. The values of $a_{i j}$ 's are listed in Table 1 .

From this principle, we formalize the Relatively Judgment Matrix as follows.

$$
\mathrm{A}=\left(a_{i j}\right)_{m \times m}=\left[\begin{array}{cccc}
a_{11} & a_{12} & \cdots & a_{1 m} \\
a_{21} & a_{22} & \cdots & a_{2 m} \\
\vdots & \vdots & \ddots & \vdots \\
a_{n 1} & a_{n 2} & \cdots & a_{m m}
\end{array}\right]
$$

The basic steps of the fuzzy AHP modeling are given as follows [6].

\section{Step 1: Constructing a fuzzy judgment matrix}

For representing uncertain results of comparison between two criteria provided by experts, a $\operatorname{TFN}(I, m, u)$ is used. This fuzzy number is characterized by the median $m$, and the upper and lower bounds $u$ and $I$. The upper bound and lower bound, respectively, represent the highest and lowest scores recognized by the experts.

For instance, by comparing the criteria 1 and 2, one expert gives $\operatorname{TFN}(0.5,1,1.5)$. Here, the median $=1$ represents that the importance weight of the criterion 2 is almost the same as that of the criterion 1 for this expert. Also, the relative importance weight of these two criteria varies between 0.5 and 1.5 .

When introducing TFNs into the results of comparison between the $m$ evaluation criteria, the Eq. (1) can be then transformed into the form Eq. (2) as follows:

$$
\tilde{A}=\left(\tilde{a}_{i j}\right)_{m \times m}=\left[\begin{array}{cccc} 
& & & \\
\tilde{a}_{11} & \tilde{a}_{12} & \cdots & \tilde{a}_{1 m} \\
\tilde{a}_{21} & \tilde{a}_{22} & \cdots & \tilde{a}_{2 m} \\
\vdots & \vdots & \ddots & \vdots \\
\tilde{a}_{m 1} & \tilde{a}_{m 2} & \cdots & \tilde{a}_{m m}
\end{array}\right]
$$

where $\quad \tilde{A}$ is the TFN $\tilde{a}_{i j}=\operatorname{TFN}\left(l_{i j}, m_{i j}, u_{i j}\right)$, $\tilde{a}_{j i}=T F N\left(\frac{1}{u_{i j}}, \frac{1}{m_{i j}}, \frac{1}{l_{i j}}\right)$
Step 2: Determine the value of fuzzy synthetic extent [7]

Let $M_{i}$ be the comprehensive fuzzy value of the evaluation criterion $c_{i}$ of the hierarchical structure in Figure 2. The calculation method is given as follows:

$M_{i}=\sum_{j=1}^{m} \tilde{a}_{i j} \div\left(\sum_{i=1}^{m} \sum_{j=1}^{m} \tilde{a}_{i j}\right), i=1,2, \cdots, m$

where $\tilde{a}_{\mathrm{ij}}$ is the ith row and $j$ th column element of the fuzzy judgment matrix.

Step 3: Computation of the weights of the evaluation criteria

Let us assume that we have two evaluation criteria $c_{i}$ and $c_{j}$ whose TFNs are $M_{i}\left(l_{i}, m_{i}, u_{i}\right)$ and $M_{j}\left(l_{j}, m_{j}, u_{j}\right) \cdot M_{i} \square M_{j}$ is also a TFN whose membership function is given by the following expression:

$$
\mathrm{V}\left(M_{i} \geq M_{j}\right)=\sup _{x \geq y}\left[\min \left(u_{M_{i}}(x), u_{M_{j}}(y)\right)\right]
$$

We propose that $\left.M_{i} \geq M_{j}\right)=u(\mathrm{~d})$, d is the horizontal coordinate of the intersection between $M_{i}$ and $M_{j}$, and

$u(\mathrm{~d})=\left\{\begin{array}{cc}1 & m_{i} \geq m_{j} \\ \frac{l_{j}-u_{i}}{\left(m_{i}-u_{i}\right)-\left(m_{j}-l_{j}\right)} & m_{i} \leq m_{j}, \quad u_{i} \geq l_{j} \\ 0 & \text { otherwise }\end{array}\right.$

The fact that the TFN $M_{i}$, associated with the criterion $c_{i}$, is larger than the TFNs of the other $m-1$ criteria can be defined as:

$V\left(M_{i} \geq M_{1}, M_{2}, \cdots M_{m}\right)=\min \mathrm{V}\left(M_{i} \geq M_{k}\right)=w^{\prime}\left(c_{i}\right)$ for $\mathrm{k}=1,2, \cdots \mathrm{m}$ and $\mathrm{k} \neq \mathrm{i}$.

This result represents the possibility with which the fuzzy number $M_{i}$ is greater than all the others $M_{1}, M_{2}, \ldots, M_{m}$ . It represents the relative preference or weight of the criterion $c_{i}$ over the others. The final weights of the criteria $c_{1}, \ldots, c_{m}$, denoted as $w\left(c_{i}\right)$ 's, are obtained by normalizing the values of $w^{\prime}\left(c_{i}\right)$ 's for all $i$.

\subsection{Mathematical formalization}

To model this relationship and perform the concerned analysis, we present the mathematical formalization of the different categories of variables.

Category 1: Let $\mathrm{BS}=\left\{\mathrm{bs}_{1}, \ldots, \mathrm{bs}_{m}\right\}$ be a set of $m(m=20)$ body shapes, representing all the combinations of the 5 standard tall-low types and 4 fat-thin types obtained from the Chinese National Standard GB/T 1335.2-1997, i.e., "X1XY1," "X2XY1," "X3XY1," "X4XY1," "X5xY1," "X1xY2," "X2XY2," "X3XY2," "X4XY2," "X5XY2," "X1XY3," "X2XY3," "X3XY3," "X4XY3," "X5xY3," “X1xY4," “X2xY4," “X3XY4," "X4xY4," and "X5xY4." 
Category 2: Let $\mathrm{S}=\left\{\mathrm{S}_{1}, \ldots, \mathrm{S}_{n}\right\}$ be a set of $n$ style keywords $(n=8)$, including "Elegant," "Feminine," "Young," "Sexy," "Classic," "Romantic," "Folk," and "Sport."

Category 3: Let $\mathrm{C}=\left\{\mathrm{c}_{1} \ldots \mathrm{c}_{k}\right\}$ be a set of $k$ visual images $(k=6)$.

Category 4: Let $I=\left\{I_{1}, \ldots, I_{q}\right\}$ be a set of $q$ weights $(q=3)$ corresponding to the three input parts BS, S, and $C$. These weights are obtained by human evaluations using the fuzzy AHP method.

Category 5: Let CP be a profile of a specific consumer including the body shape, style keywords, and visual images. It is expressed by an $\mathrm{N}$-dimensional weighted vector denoted as

$$
\mathrm{CP}=\left(I_{1} \times \mathrm{bs}_{1}, \ldots, I_{1} \times \mathrm{bs}_{m}, I_{2} \times \mathrm{s}_{1}, \ldots, I_{2} \times \mathrm{s}_{n}, I_{3} \times \mathrm{c}_{1}, \ldots, I_{3}\right.
$$
$\times\left(C_{k}\right)$.

\section{Implementation of consumer profile}

\subsection{Fuzzy description of height}

Usually, we choose two indices to describe the shape of the human body, namely tall-low and fat-thin [8]. However, in practice, the question of how to efficiently evaluate "tall-low" and "fat-thin" is quite a vague one for all consumers, designers, and shoppers. Taking this observation into consideration, we use fuzzy sets to express human body shapes.

Based on the garment designers' knowledge and experience, "tall-low" can be expressed by b1 (stature), and "fat-thin" by b1 and b4 (weight). We can describe the tall-low as five levels: X1: short, X2: a little short, X3: middle, X4: a little tall, and X5: tall.

\subsection{Fuzzy membership functions}

In the application of fuzzy theory, the first problem is to establish fuzzy membership functions of fuzzy sets. For fuzzy sets, the membership functions not only reflect the characteristics of the fuzzy concept but also can achieve mathematical operations and processing.

The fuzzy membership functions and rules are obtained by evenly dividing the entire range of the tall-low body data into 5 fuzzy values or 5 classes, denoted as X1, X2, X3, X4, and X5 (see Figure 3).

In the definition of the fuzzy membership function, X1 and $\mathrm{X} 5$ represent the minimal and maximal fuzzy values (two extremes) of the height $b 1$, corresponding to $\operatorname{Min}\{b 1 i \mid i=1, \ldots$, $\mathrm{p}\}=145 \mathrm{~cm}$ and $\operatorname{Max}\{\mathrm{b} 1|i| i=1, \ldots, p\}=175 \mathrm{~cm}$, respectively. The medium fuzzy value $X 3$ corresponds to the standard size $160 \mathrm{~A}$ with a height of $160 \mathrm{~cm} . X 2$ and $X 4$ are centered on the middle point of X1, X3 (i.e., $(145+160) / 2=152.5 \mathrm{~cm})$ and that of $X 3, X 5$ (i.e. $(175+160) / 2=167.5 \mathrm{~cm})$, respectively.

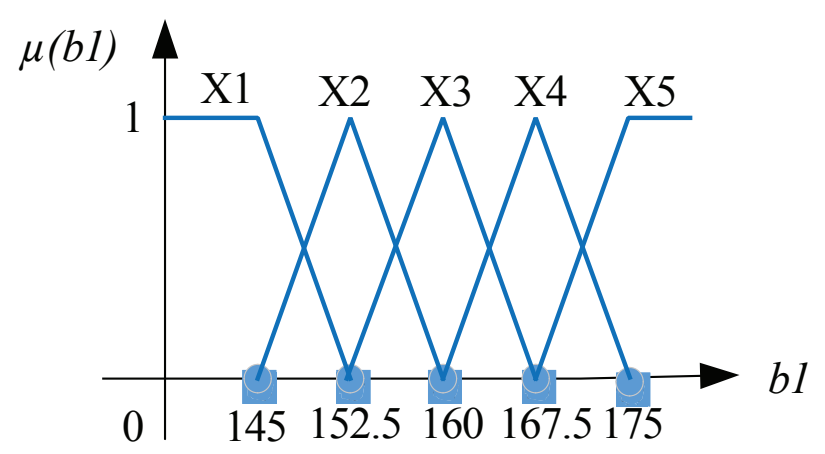

Figure 3. Fuzzy membership functions (tall-low).

Centered on these key points, the fuzzy membership functions of (tall-low) are defined by the following expressions:

$$
\mu(b 1)=\left\{\begin{array}{cc}
X 1=1 & b 1 \leq 145 \\
X 1=-0.133 b 1+20.33 & b 1>145 \text { and } b 1 \leq 152.5 \\
X 1=0 & \text { otherwise }
\end{array}\right.
$$

$$
\mu(b 1)=\left\{\begin{array}{rr}
X 2=0.133 b 1-19.33 & b 1>145 \text { and } b 1 \leq 152.5 \\
X 2=-0.133 b 1+21.33 & b 1>152.5 \text { and } b 1 \leq 160 \\
X 2=0 & \text { otherwise }
\end{array}\right.
$$

$$
\mu(b 1)=\left\{\begin{array}{rr}
X 3=0.133 b 1-20.33 & b 1>152.5 \text { and } b 1 \leq 160 \\
X 3=-0.133 b 1+22.33 & b 1>160 \text { and } b 1 \leq 167.5 \\
X 3=0 & \text { otherwise }
\end{array}\right.
$$

$$
\mu(b 1)=\left\{\begin{array}{cc}
X 4=0.133 b 1-21.33, & b 1>160 \text { and } b 1 \leq 167.5 \\
X 4=-0.133 b 1+23.33 & b 1>167.5 \text { and } b 1 \leq 175 \\
X 4=0 & \text { otherwise }
\end{array}\right.
$$

$$
\mu(b 1)=\left\{\begin{array}{cc}
X 5=1 & b 1 \geq 175 \\
X 5=0.133 b 1-22.33 & b 1>167.5 \text { and } b 1 \leq 175 \\
X 5=0 & \text { otherwise }
\end{array}\right.
$$

Based on the fuzzy membership functions (tall-low) described above, consumer's body data can be easily converted to fuzzy or linguistic values for further processing.

Example: For a given consumer with $\mathrm{b} 1=163 \mathrm{~cm}$, by using these fuzzy memberships functions, we can obtain the corresponding fuzzy value as follows: $(X 1, X 2, X 3, X 4, X 5)=(0,0,0.65,0.35,0)$

\subsection{Fuzzy description of "fat and thin"}

The fuzzy membership function of "fat and thin" can be expressed by the value of body mass index (BMI).

The BMI is a value derived from the weight and height of an individual [9]. The BMI is defined as the body weight divided by the square of the body height and is universally expressed in units of $\mathrm{kg} / \mathrm{m}^{2}$, resulting from weight in kilograms and height in meters. In this paper, we have $B M I=b 4 /(b 1 / 100)^{2}$.

The basis of BMI was designed by Adolphe Quetelet from 1830 to 1850 during which he developed what he called "social 
physics." The modern term BMI for the ratio of human body weight to squared height was coined in a paper published in the July 1972 edition of the Journal of Chronic Diseases by Ancel Keys [10].

BMI provides a simple digital measure of a person's thickness and thinness, allowing health professionals to discuss weight problems more objectively with patients. BMI was designed to be used as a simple method for categorizing average sedentary (physically inactive) populations with an average body composition [11].

$\mathrm{BMI}$ is a measure of body fat based on height and weight and is suitable for both men and women aged 18-65 years. The range of all the BMI values has been divided into different categories of health states as follows (Table 2). [12]

These recommended distinctions along the linear scale may differ from country to country and time to time, making global, longitudinal surveys problematic.

\subsection{Fuzzy membership functions (fat and thin)}

This paper is based on the above BMI values given by Hong Kong, Japan, and Singapore because their body shapes, health states, and eating habits are similar to those of the Chinese population. The fuzzy membership functions and rules are obtained by evenly dividing the entire range of BMI or fatthin, calculated from the body data b1 and b4, into 4 classes or 4 fuzzy values denoted as Y1, Y2, Y3, and Y4 (see Figure 4).

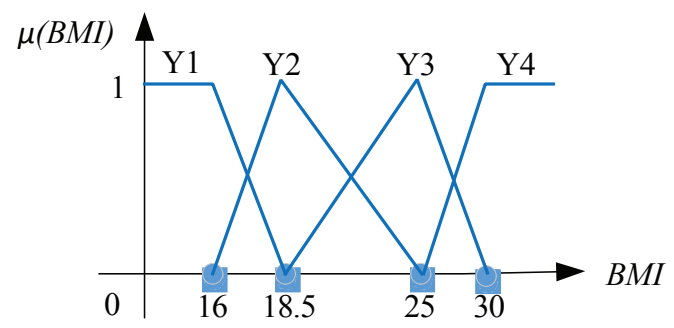

Figure 4. Fuzzy membership functions of (fat-thin).

$$
\mu(B M I)=\left\{\begin{array}{cr}
Y 1=1 & B M I \leq 16 \\
Y 1=-0.4 B M I+7.4 & B M I>16 \text { and } B M I \leq 18.5 \\
Y 1=0 & \text { otherwise }
\end{array}\right.
$$

Table 2. Ranges of BMI values given by WTO.

\begin{tabular}{|c|c|c|}
\hline Category & \multicolumn{2}{|c|}{ BMI $\left(\mathbf{k g} / \mathbf{m}^{2}\right)$} \\
\hline Very severely underweight & From & To \\
\hline Severely underweight & 15 & 15.0 \\
\hline Underweight & 16 & 16 \\
\hline Normal (healthy weight) & 18.5 & 25 \\
\hline Overweight & 25 & 30 \\
\hline Obese Class I (moderately obese) & 30 & 35 \\
\hline Obese Class II (severely obese) & 35 & 40 \\
\hline Obese Class III (very severely obese) & 40 & \\
\hline
\end{tabular}

$$
\mu(B M I)=\left\{\begin{array}{cc}
Y 2=0.4 B M I-6.4 & B M I>16 \text { and } B M I \leq 18.5 \\
Y 2=-0.15 B M I+3.8 & B M I>18.5 \text { and } B M I \leq 25 \\
Y 2=0 & \text { otherwise }
\end{array}\right.
$$

$$
\mu(B M I)=\left\{\begin{array}{cr}
Y 3=0.15 B M I-2.8 & B M I>18.5 \text { and } B M I \leq 25 \\
Y 3=-0.2 B M I+6 & B M I>25 \text { and } B M I \leq 30 \\
Y 3=0 & \text { otherwise }
\end{array}\right.
$$

$\mu(B M I)=\left\{\begin{array}{lr}Y 4=1 & B M I>30 \\ Y 4=0.2 B M I-5 & B M I>25 \text { and } B M I \leq 30 \\ Y 4=0 & \text { otherwise }\end{array}\right.$

Based on the previous definition of the fuzzy membership functions of BMI (fat-thin), the input personal data of a particular consumer can be easily converted into a corresponding fuzzy fat-thin value. An example is given below.

For the same female consumer (b1=163 cm and b4=50 kg) previously presented, we obtain $\mathrm{BMI}=18.8$. By using the previous fuzzy membership functions of (fat-thin), we obtain the corresponding fuzzy value as follows.

$(\mathrm{Y} 1, \mathrm{Y} 2, \mathrm{Y} 3, \mathrm{Y} 4)=(0,0.98,0.02,0)$

This means that the index of fat-thin is between $\mathrm{Y} 2$ and $\mathrm{Y} 3$ and closer to Y2.

\section{A real case}

\subsection{Application of the FAHP algorithm}

For a specific consumer, we consider that her profile is composed of three parts of information: K1 - body data (b1, b2, b3, b4), K2 - style keywords, and K3 - visual images.

The fuzzy evaluation matrix given by the three experts for pairwise comparisons of these three parts is given as follows (see Table 3):

These three pairwise comparisons given by the three experts can be aggregated into unified TFNs by using classical fuzzy operations. We take $\tilde{a}_{21}$ (comparison of K2 and K1) as an example:

$(1 / 3+1 / 4+1 / 2) / 3=0.36$

$(1 / 2+1 / 3+1 / 1) / 3=0.61$

$(1 / 1+1 / 2+1 / 1) / 3=0.83$

Then, the final fuzzy number of $\tilde{a}_{21}$ is $\operatorname{TFN}(0.36,0.61,0.83)$.

The final fuzzy matrix $\tilde{A}=\left(\tilde{a}_{i j}\right)_{3.3}$ representing the aggregated pairwise comparisons is given in Table 4 . 
Table 3. The pairwise comparisons of the three input parts given by the three experts and expressed by TFN fuzzy numbers.

\begin{tabular}{|c|c|c|c|}
\hline & K1 & K2 & K3 \\
\hline $\mathrm{K} 1$ & $\begin{array}{l}\text { TFN }(1,1,1) \\
\text { TFN }(1,1,1) \\
\text { TFN }(1,1,1)\end{array}$ & $\begin{array}{l}\text { TFN }(1,2,3) \\
\text { TFN }(2,3,4) \\
\text { TFN }(1,1,2)\end{array}$ & $\begin{array}{l}\operatorname{TFN}(1,1,2) \\
\operatorname{TFN}(1,1,2) \\
\operatorname{TFN}(1,2,3)\end{array}$ \\
\hline K2 & $\begin{array}{l}\text { TFN }(1 / 3,1 / 2,1 / 1) \\
\operatorname{TFN}(1 / 4,1 / 3,1 / 2) \\
\operatorname{TFN}(1 / 2,1 / 1,1 / 1)\end{array}$ & $\begin{array}{l}\operatorname{TFN}(1,1,1) \\
\operatorname{TFN}(1,1,1) \\
\operatorname{TFN}(1,1,1)\end{array}$ & $\begin{array}{l}\operatorname{TFN}(1,1,2) \\
\operatorname{TFN}(1,2,3) \\
\operatorname{TFN}(1,1,2)\end{array}$ \\
\hline K3 & $\begin{array}{l}\text { TFN }(1 / 2,1 / 1,1 / 1) \\
\operatorname{TFN}(1 / 2,1 / 1,1 / 1) \\
\operatorname{TFN}(1 / 3,1 / 2,1 / 1)\end{array}$ & $\begin{array}{l}\operatorname{TFN}(1 / 2,1 / 1,1 / 1) \\
\operatorname{TFN}(1 / 3,1 / 2,1 / 1) \\
\operatorname{TFN}(1 / 2,1 / 1,1 / 1)\end{array}$ & $\begin{array}{l}\operatorname{TFN}(1,1,1) \\
\operatorname{TFN}(1,1,1) \\
\operatorname{TFN}(1,1,1)\end{array}$ \\
\hline
\end{tabular}

The comprehensive weight of each criterion $K_{i}$ can be calculated according to the general principle of the FAHP method.

Step 1: Determine the value of fuzzy synthetic extent.

Let $M_{i}$ be the comprehensive fuzzy value of the evaluation criterion $K_{i}$ of the hierarchical structure in Table 4 . The calculation method is given as follows:

$$
M_{i}=\sum_{j=1}^{m} \tilde{a}_{i j} \div\left(\sum_{i=1}^{m} \sum_{j=1}^{m} \tilde{a}_{i j}\right), i=1,2, \cdots, m
$$

where $\tilde{a}_{\mathrm{ij}}$ is the element at the ith row and jth column of the fuzzy judgment matrix.

The calculation steps are given as follows:

$\sum_{i=1}^{3} \sum_{j=1}^{3} \tilde{a}_{i j}=(1+1+1)+(1.33+2+3)+\cdots+(1+1+1)$

We have:

$\sum_{j=1}^{3} \tilde{a}_{1 j}=(1+1+1)+(1.33+2+3)+(1+1.33+2.33)$

$\sum_{j=1}^{3} \tilde{a}_{2 j}=(0.36+0.61+0.83)+(1+1+1)+(1+1.33+2.33)$

$\sum_{j=1}^{3} \tilde{a}_{3 j}=(0.44+0.83+1)+(0.44+0.83+1)+(1+1+1)$

We have:

$$
\begin{aligned}
& M_{1}=\sum_{j=1}^{3} \tilde{a}_{1 j} \div\left(\sum_{i=1}^{3} \sum_{j=1}^{3} \tilde{a}_{i j}\right), i=1,2,3 \\
& M_{2}=\sum_{j=1}^{3} \tilde{a}_{2 j} \div\left(\sum_{i=1}^{3} \sum_{j=1}^{3} \tilde{a}_{i j}\right), i=1,2,3 \\
& M_{3}=\sum_{j=1}^{3} \tilde{a}_{3 j} \div\left(\sum_{i=1}^{3} \sum_{j=1}^{3} \tilde{a}_{i j}\right), i=1,2,3
\end{aligned}
$$

Step 2: Computation of the weights of the evaluation criteria

$M_{i} \geq M_{j}$ is also a TFN whose membership function is given by the following expressions:

$$
\mathrm{v}\left(M_{1} \geq M_{2}\right)=\sup _{x \geq y}\left[\min \left(u_{M_{1}}(x), u_{M_{2}}(y)\right)\right]
$$$$
\mathrm{V}\left(M_{1} \geq M_{2}\right)=u(\mathrm{~d})=\left\{\begin{array}{cl}
1 & m_{1} \geq m_{2} \\
\frac{l_{2}-u_{1}}{\left(m_{1}-u_{1}\right)-\left(m_{2}-l_{2}\right)} & m_{1} \leq m_{2}, \quad u_{1} \geq l_{2} \\
0 & \text { otherwise }
\end{array}\right.
$$

The TFN $M_{i}$, associated with the criterion $K_{i}$ is larger than the TFNs of the other $m-1$ criteria and can be defined as:

$V\left(M_{i} \geq M_{1}, M_{2}, \cdots M_{m}\right)=\min \mathrm{V}\left(M_{i} \geq M_{k}\right)=w^{\prime}\left(K_{i}\right)$ for $\mathrm{k}=1,2, \cdots \mathrm{m}$ and $\mathrm{k} \neq \mathrm{i}$.

So, the weights $\mathrm{I} 1, \mathrm{I} 2$, and $\mathrm{I} 3$ associated with $\mathrm{K} 1, \mathrm{~K} 2$, and $\mathrm{K} 3$ are calculated as follows:

$\mathrm{I} 1=\min \mathrm{V}\left(M_{1} \geq M_{2}, M_{3}\right)$

$12=\min \mathrm{V}\left(M_{2} \geq M_{1}, M_{3}\right)$

I3=min $\mathrm{V}\left(M_{3} \geq M_{1}, M_{2}\right)$

Table 4. The final fuzzy judgment matrix.

\begin{tabular}{|c|c|c|c|}
\hline & K1 & K2 & K3 \\
\hline K1 & $\operatorname{TFN}(1,1,1)$ & $\operatorname{TFN}(1.33,2,3)$ & $\operatorname{TFN}(1,1.33,2.33)$ \\
\hline K2 & $\operatorname{TFN}(0.36,0.61,0.83)$ & $\operatorname{TFN}(1,1,1)$ & $\operatorname{TFN}(1,1.33,2.33)$ \\
\hline K3 & $\operatorname{TFN}(0.44,0.83,1)$ & $\operatorname{TFN}(0.44,0.83,1)$ & $\operatorname{TFN}(1,1,1)$ \\
\hline
\end{tabular}


We normalize I1, I2, and I3 so that their sum is 1 and obtain the following final weight of each index:

$$
\left(\frac{I_{1}}{I_{1}+I_{2}+I_{3}}, \frac{I_{2}}{I_{1}+I_{2}+I_{3}}, \frac{I_{3}}{I_{1}+I_{2}+I_{3}}\right) \approx(0.48,0.31,0.21)
$$

\subsection{Fuzzy comprehensive evaluation method}

We combine the three parts of information (body data, style keywords, and visual images) by using the FCEM [13] and considering their respective weights, 11,12 , and 13 , calculated previously.

The consumer profile is obtained by FCEM. It is denoted as $\mathrm{CP}=\left(I_{1} \times \mathrm{bs}_{1}, \ldots, I_{1} \times \mathrm{bs}_{m}, I_{2} \times \mathrm{s} 1, \ldots, I_{2} \times \mathrm{s}_{n}, I_{3} \times \mathrm{c}_{1}, \ldots, I_{3}\right.$ $\times\left(\mathrm{C}_{k}\right)$.

\subsection{A real case of consumer profile}

Here is a real case to illustrate the performance of the proposed method. For a given consumer, the data include the following three parts:

(1) Body data: b1=163 cm; b2=104 cm; b3=92 cm; b4=50 kg.

(2) Style keywords: s3 (young).

(3) Preferred image: c6 (picture 6).

In the previous paragraphs, we calculated the corresponding fuzzy sets from the body data, describing the criteria of (talllow) and (fat-thin), i.e., $(X 1, X 2, X 3, X 4, X 5)=(0,0,0.65,0.35,0)$ and $(Y 1, Y 2, Y 3, Y 4)=(0,0.98,0.02,0)$.

Next, we calculate the BS, S, and C based on the three parts of the input data.

|(1) $B S=\{0,0,0,0,0,0,0,0.64,0.34,0,0,0,0.01,0.01,0,0,0,0,0,0\}$, representing $m \quad(m=20)$ body shapes, formed by the combinations of multiplication of all elements "XixYj" $(i=1, \ldots, 5$ and $j=1, \ldots, 4)$ in "tall-low" and "fat-thin."

(2) $S=\{0,0.5,0,0.5,0,0,0,0\}$, representing that the consumer chooses the 2 nd and 4th style elements in the same time from all the $n$ styles keywords $(n=8)$. The selected keywords are "Feminine" and "Sexy," respectively. The 2nd and 4th elements of $S$ are 0.5 and the others 0 so that the sum of all elements in $S$ is 1 . If only "young" is selected, we have $S=\{0,0,1,0,0,0,0,0\}$. For simplicity, we just suppose that this consumer selects "young."

The elements of $\mathrm{S}$ can be defined by the consumer herself. For example, we can give $S=\{0.25,0.25,0,0.3,0,0.2,0,0\}$, showing $25 \%$ for "Elegant," $25 \%$ for "Feminine," $30 \%$ for "Sexy," and $20 \%$ for "Romantic."
(3) $C=\{0,0,0,0,0,1\}$, representing that the consumer selects "picture 6" from all the $k$ visual images $(k=6)$. The elements of $\mathrm{C}$ can be defined by the consumer herself.

We can obtain $\left(I_{1}, I_{2}, I_{3}\right)=(0.48,0.31,0.21)$, and the body size for this consumer is 165B; then we can calculate the consumer profile by the following expression:

$$
\begin{aligned}
& \mathrm{CP}=\left(I_{1} \times \mathrm{bs}_{1}, \ldots, I_{1} \times \mathrm{bs}_{m}, I_{2} \times \mathrm{s} 1, \ldots, I_{2} \times \mathrm{s}_{n}, I_{3} \times \mathrm{c} 1, \ldots, I_{3}\right. \\
& \left.\times \mathrm{C}_{k}\right) \\
& =(0,0,0,0,0,0,0,0.31,0.16,0, \quad 0,0,0.005,0.005,0, \quad 0,0,0,0,0, \\
& 0,0,0.31,0,0,0,0,0,0,0,0,0,0,0.21) .
\end{aligned}
$$

\section{CONCLUSION}

We first define the consumer profile that will be used as basis in a consumer-oriented recommendation system [14]. Next, we give the mathematical formalization of the concerned concepts and model of the body shapes, style keywords, and visual images, and the acquired data are processed by using fuzzy sets, fuzzy composition operations, and the fuzzy AHP algorithm.

Fuzzy techniques are the main computational tool used in this paper because they are more relevant to modeling and analysis of data acquired. In fact, the evaluation data on body shapes (e.g., description of tall-low and fat-thin), style keywords, and visual images can never be accurately expressed. The determination of weights for the three inputs of the proposed recommendation system, i.e., body shapes, style keywords, and visual images, is performed using fuzzy AHP, permitting to effectively deal with the subjectivity of evaluation criteria related to human judgments. The FCEM, leading to an efficient general evaluation with a variety of criteria, is also successfully applied for aggregating the data from the three inputs of the recommendation system to form a relevant consumer profile.

The method of building the consumer profile can be widely used for a consumer-oriented recommendation system. It can guide shoppers and manufactures to recommend more competitive garments in the consumer-oriented market.

Compared to other existing methods, the way to name the consumer profile is more robust and powerful due to its capacity to handle body shapes, style keywords, and visual images. This work can be further extended to support other fashion products such as suits, shoes, accessories, and more.

Due to time constraints, the current work is far from perfect. In future work, we expect that our focus will be on the following aspects:

(1) In the future, to obtain more generalized and concrete information about the consumer profile, ontology technology must be integrated into the consumer profile.

(2) In the future, more complicated strategies can be introduced to make the body shapes more accurate and robust. 


\section{References}

[1] PROFILES, B. C. (2001). Using data mining methods to build customer profiles.

[2] Zhang, J., Zeng, X., Liu, K., Yan, H., Dong, M. (2008). Jeans knowledge base development based on sensory evaluation technology for customers' personalized recommendation. International Journal of Clothing Science \& Technology, 30(1), 101-111.

[3] Sun, M., Li, T., Ji, B., Jiao, Y., Tang, S. (2012). Evaluation research on assessment of clinical nursing teaching quality based on fuzzy comprehensive evaluation method. Journal of Convergence Information Technology, 7(8), 82-91.

[4] Civanlar, M. R., Trussell, H. J. (1986). Constructing membership functions using statistical data. Fuzzy sets and Systems, 18(1), 1-13.

[5] Kutlu, A. C., Ekmekçioğlu, M. (2012). Fuzzy failure modes and effects analysis by using fuzzy TOPSIS-based fuzzy AHP. Expert Systems with Applications, 39(1), 61-67.

[6] Zhu, Y., Ruan, D., Zeng, X., Koehl, L., Chaigneau, C. (2010). Characterization of fashion themes using fuzzy techniques for designing new human centered products. International Journal of Computational Intelligence Systems, 3(4), $p$. 452-460.

[7] Lijin, Z. K. Z. X. X. (1997). The method and applications of fuzzy AHP. Systems Engineering - -Theory \& Practice, 12.

[8] Zhang, J., Zeng, X., Koehl, L., Dong, M. (2016). Consumer- oriented intelligent garment recommendation system. In Uncertainty Modelling in Knowledge Engineering and Decision Making: Proceedings of the 12th International FLINS Conference. World Scientific.

[9] Kuczmarski, M. F., Kuczmarski, R. J., Najjar, M. (2001). Effects of age on validity of self-reported height, weight, and body mass index: Findings from the Third National Health and Nutrition Examination Survey, 1988-1994. Journal of the American Dietetic Association, 101(1), 2834.

[10] Eknoyan, G. (2008). Adolphe Quetelet (1796-1874)—the average man and indices of obesity. Nephrology Dialysis Transplantation, 23(1), 47-51.

[11] Baladad, B. M. S., Magsombol, J. V., Roxas, J. N. B., De castro, E. L., Dolot, J. A. (2016). Development of automated body mass index calculation device. International Journal of Applied Engineering Research, 11(7), 5195-5201.

[12] World Health Organization. (2006). Global database on body mass index.[13] Feng, S., Xu, L. (1999). An intelligent decision support system for fuzzy comprehensive evaluation of urban development. Expert Systems with Applications, 16(1), 21-32.

[14] Zhang, J., Zeng, X., Koehl, L., Dong, M. (2018). Recommending garment products in E-shopping environment by exploiting an evolutionary knowledge base. International Journal of Computational Intelligence Systems, 11(1), 340-354. 